\title{
The modern concept of the INCA project elements
}

\author{
V.V. Ammosov ${ }^{a}$, G.I. Britvich ${ }^{a}$, A.P. Chubenko ${ }^{b}$, P.A. Chubenko ${ }^{b}$, V.I. Drobzhev ${ }^{c}$, \\ A.D. Erlykin ${ }^{b}$, G.I. Kol'tsov ${ }^{d}$, O.N.Kryakunova ${ }^{c}$, S.V.Kryukov ${ }^{c}$, G.I. Merzon ${ }^{b}$, \\ B.N. Mukashev ${ }^{e}$, K.K. Mukashev ${ }^{f}$, R.A. Mukhamedshin ${ }^{g}$, V.N. Murashev ${ }^{d}$, \\ M.I. Panasyuk ${ }^{h}$, V.P. Pavlyuchenko ${ }^{b}$, S.A. Petrochenkov ${ }^{i}$, V.A. Ryabov ${ }^{b}$, \\ O.G. Ryazhskaya ${ }^{g}$, T. Saito ${ }^{j}$, A.L. Shchepetov ${ }^{b}$, N.M. Sobolevskii ${ }^{g}$, A.P. Soldatov ${ }^{a}$, \\ V.M. Sultangazin ${ }^{k}$, I.S. Trostin ${ }^{l}$, V.G. Vasil' chenko ${ }^{a}$, G.T. Zatsepin ${ }^{g}$, and A.P. Zhukov ${ }^{g}$ \\ (a) Institute for High-Energy Physics, Protvino, 142284 Russia \\ (b) Lebedev Physical Institute, Moscow, 119991 Russia \\ (c) Ionosphere Institute, Almaty, Kazakhstan Republic \\ (d) Moscow State Institute of Steel and Alloys, Moscow, 117936 Russia \\ (e) Physical-Technnical Institute,Almaty, Kazakhstan Republic \\ (f) Abai Almaty State University, Almaty, Kazakhstan Republic \\ (g) Institute for Nuclear Research, Moscow, 117312 Russia \\ (h) Skobel'tsyn Institute of Nuclear Physics, Moscow, 119992 Russia \\ (i) Joint Institute for Nuclear Research, Dubna, 141980 Russia \\ (j) Institute for Advanced Studies, 1-29-6 Shinjuku, Tokyo, 162-0022 Japan \\ (k) Institute for Space Research, Almaty, Kazakhstan Republic \\ (l) Institute for Theoretical and Experimental Physics, Moscow, 117259 Russia \\ Presenter: R.A. Mukhamedshin (muhamed@sci.lebedev.ru), rus-mukhamedshin-R-abs2-og15-poster
}

The modern concept of elements of a device designed to study the spectrum and composition of the primary cosmic radiation up to energies of $\sim 10^{16} \mathrm{eV}$ and based on new technologies is considered.

\section{Introduction}

The main goal of the INCA project [1] is the design of a multipurpose spectrometer for fundamental studies of the primary cosmic radiation (PCR), in part, for measurements of the spectrum and composition of PCR components at "knee" energies up to $\sim 10^{16} \mathrm{eV}$. The project employs a new-type device, namely, ionizationneutron calorimeter (INCA), measuring both ionization and neutron signals and exploiting bipolar pixel siliconbased detectors developed in the framework of this project.

\section{Properties of INCA}

General Properties. Basic characteristics of the current INCA conception are listed in Tables $1-3$ and schematically shown in Figs. $1-4$. A layout of INCA is shown in Fig. 1. The INCA's exterior consists of two layers ( $A$ and $B$ ) separated with a gap (Figs. 1 and 3). The layer $A$ constitutes a 1-cm thick polyethylene layer containing a $1-\mathrm{mm}$ thick charge detector $(\mathrm{CD})$ divided into $5.5 \times 5.5 \mathrm{~cm}^{2}$ sections and coated by a 3-mm thick polyethylene film. The layer $B$ is made of 5 -cm thick polyethylene; PMTs and phototriodes are coming through its openings. "Helium-2" neutron counters are placed inside this layer. The interior calorimeter has 50 levels, each consisting of the alternating lead and polyethylene layers with a thickness of 1 and $29 \mathrm{~mm}$, respectively, and the layer of the long $(200 \mathrm{~cm}$ ) plastic scintillator blocks with the thickness $10 \mathrm{~mm}$ (see Fig. 2). Each of the scintillation blocks may include the light spectrum shifters (optical fibers or strips). Polyethylene and lead constitute $\sim 78 \%$ and $22 \%$ of the total weight. This is equivalent respectively to 4.3 and 0.3 in proton 
Table 1. The basic technical and scientific characteristics of the INCA device

\begin{tabular}{||l|c||}
\hline \hline \multicolumn{1}{||c||}{ Parameter } & Value \\
\hline \hline Total weight & $11050 \mathrm{~kg}$ \\
\hline Total dimensions: & $220 \times 220 \times 22 \mathrm{~cm}^{3}$ \\
Calorimeter dimensions & $200 \times 200 \times 200 \mathrm{~cm}^{3}$ \\
Thickness of the polyethylene reflector & 5 \\
Thickness of the charge detector's platform & 1 \\
Gaps between calorimeter, reflector, CD platform & 4 \\
\hline Number of scintillators $\left(5 \times 1 \times 200 \mathrm{~cm}^{3}\right)$ & 2000 \\
Number of photodetectors (PMT, phototriodes) & 4000 \\
Number of avalanche photodiodes & 400 \\
Number of silicon detector plates $\left(5.5 \times 5.5 \mathrm{~cm}{ }^{2}\right)$ & 8000 \\
\hline Number of ADC channels & 16000 \\
\hline Number of the neutron- and $\gamma$-pulse counting channels & 525 \\
\hline Power consumption & $500 \mathrm{~W}$ \\
\hline Primary information volume of registered event & $51 \mathrm{~kb}$ \\
\hline Active life time of scientific equipment & $\geq 3 \mathrm{years}$ \\
\hline Thickness in proton's mean free paths for interaction & $4.6 \lambda_{\text {int }}^{p}$ \\
Thickness in radiation lengths & $13 t_{0}$ \\
\hline Device aperture & $\sim 2.7 \pi$ \\
Geometry factor & $10-48 \mathrm{~m}^{2} \mathrm{sr}$ \\
\hline \hline
\end{tabular}

interaction paths, $\lambda_{i n t}^{p}$, or to 4 and $9 t_{0}$ in radiation lengths. Figure 3 shows the INCA's section in the plane traversing through one of the SNM17 counters. Figure 4 demonstrates a side view of one scintillator block.

Scintillators. The plastic scintillation strips are used as position-sensitive detectors of the neutron and ionization signals. PMTs, photo-triodes, and avalanche diodes are used as photodetectors. At the ends of the $80 \%$ of scintillation strips, phototriodes are placed for registering the ionization component in the amplitudemeasurement mode. Light-guide fibers passing through scintillators transmit neutron-produced single light pulses to avalanche diodes, which are used together with PMTs placed at the ends of $20 \%$ of strips for neutron registration in the pulse-counting mode. The use of difference in signal intensity at the ends of scintillator bars makes it possible to measure the cascade axis coordinates with an accuracy down to $\delta \lesssim 1 \mathrm{~mm}$ that corresponds to an angular resolution of $\sim 1^{\prime}$. The usage of $\mathrm{Cd}$ or $\mathrm{Gd}$ admixtures introducing into scintillators or coating of scintillation detectors makes it possible to increase essentially the efficiency of neutron-capture registration.

Table 2. Distribution of the INCA's power consumption

\begin{tabular}{||l|c|c||}
\hline \hline \multicolumn{1}{|c|}{ Function } & Voltage, V & Power, W \\
\hline \hline Stabilised power supply of phototriodes and neutron counters & 2000 & 50 \\
\hline Stabilised power supply of the charge detector and avalanche photo-diodes & 100 & 10 \\
\hline Power supply of the calorimeter and charge detector registration channels & 5 & 240 \\
\hline Control and data acquisition systems & - & 100 \\
\hline Power reserve & - & 100 \\
\hline Total & & 500 \\
\hline \hline
\end{tabular}


Table 3. Primary information content of registered event.

\begin{tabular}{||l|c|c|c|c||}
\hline \hline System & $\begin{array}{c}\text { Channels } \\
\text { number }\end{array}$ & $\begin{array}{c}\text { Content of data } \\
\text { per channel }\end{array}$ & $\begin{array}{c}\text { Additional } \\
\text { conditions }\end{array}$ & $\begin{array}{c}\text { Total, } \\
\mathrm{Kb}\end{array}$ \\
\hline \hline Calorimeter & 8000 & 12 bit & 2 ADC per phototriode & 12 \\
\hline Charge detector & 8000 & 12 bit & 1 ADC per section & 12 \\
\hline Pulse counting system & 525 & 1 bite & 50 bins per channel & 26 \\
\hline Monitor/control systems & - & - & - & 1 \\
\hline Total & & & & 51 \\
\hline \hline
\end{tabular}

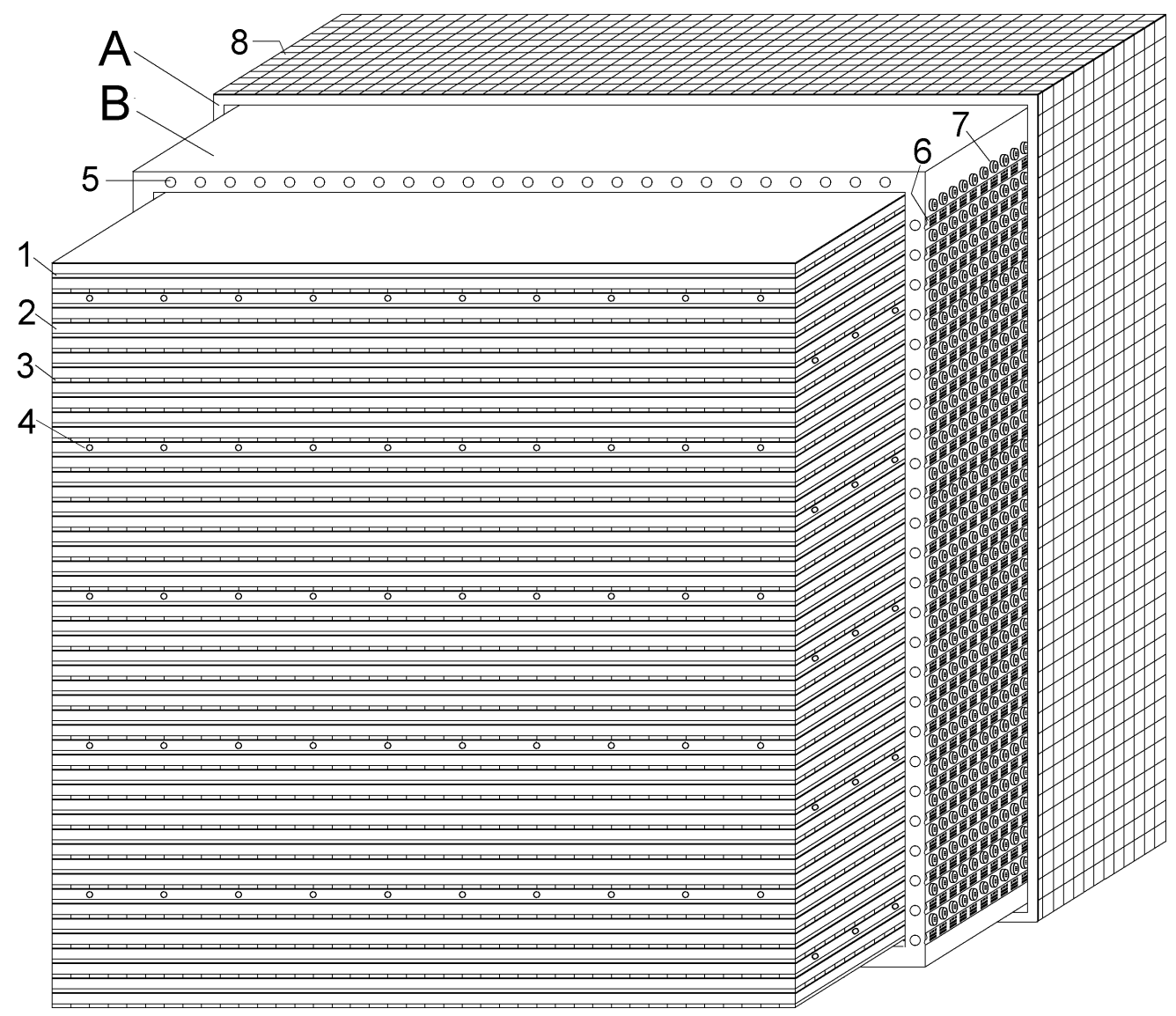

Figure 1. Layout of INCA: (1) lead; (2) polyethylene ; (3) plastic scintillators; (4) SNM-17 gas detectors; (5) Helium-2 gas detectors; (6) electronics boards; (7) photodetectors; (8) charge detectors. $A$ and $B$ are outward layers.

As scintillators detect mainly signals from delayed $\gamma$-rays produced in neutron captures and passing to relatively big distances from the point of their origin, 100 SNM17 200-cm long gas neutron counters destined to detect just neutrons are placed inside the each fifth polyethylene layer at $20 \mathrm{~cm}$ intervals.

Charge Detectors. The preferable charge detector (CD) version is a double-layer semiconductor one. However, 


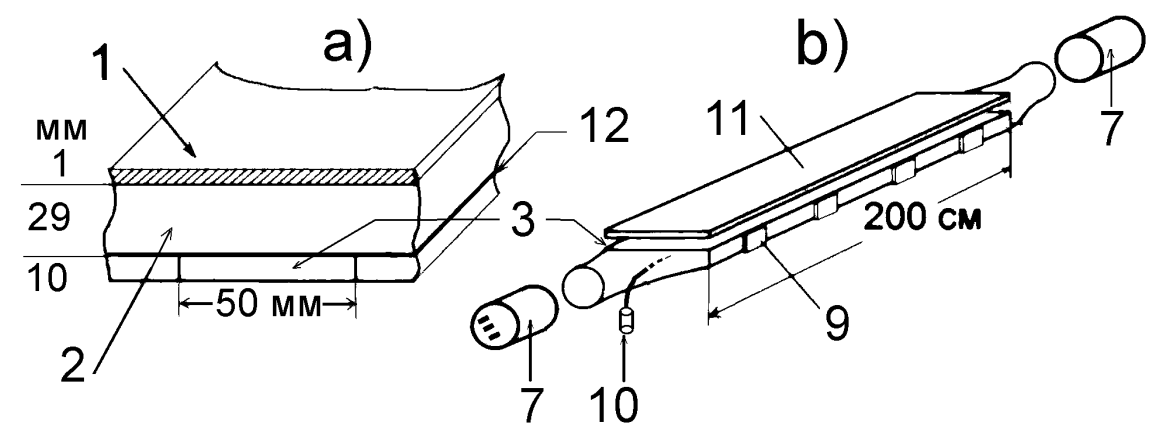

Figure 2. a) Section of one calorimeter layer; b) layout of scintillation block with photodetectors and light guides. Notation: 1) - 8) are the same as in Fig. 1; 9) photo plates; 10) fiber with avalanche photo diode; 11) wavelength-shifting light guide; 12) layer containing $\mathrm{Cd}$ or Gd. Proportions are not fulfilled.

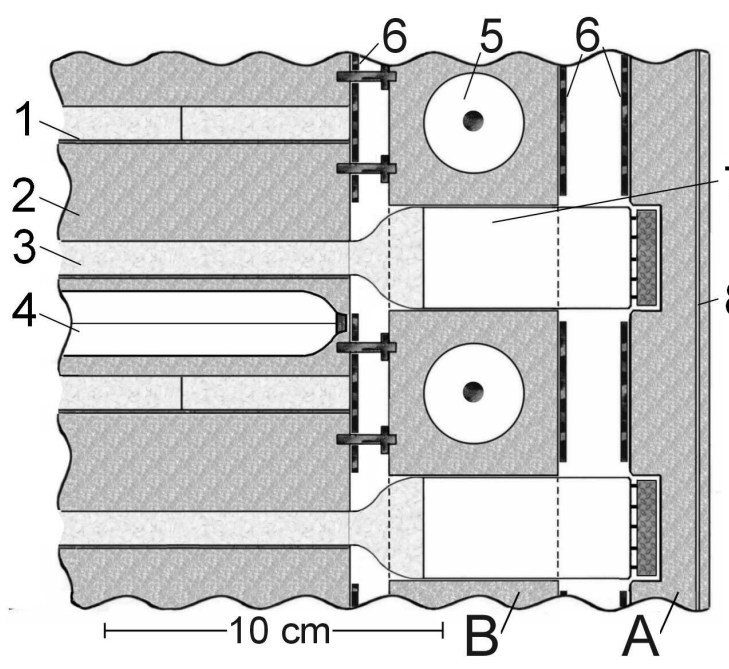

Figure 3. Cross section of scintillation unit and outer part of INCA. Notations are the same as in Figs. 1 and 2.

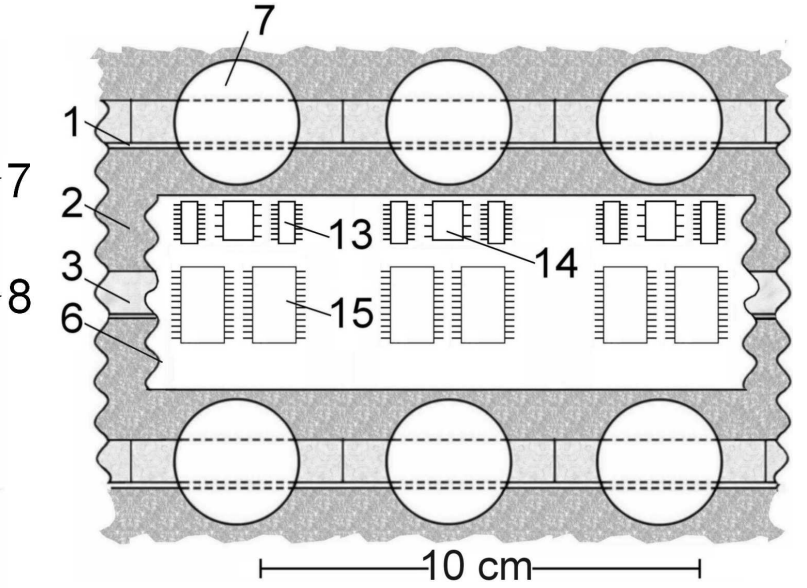

Figure 4. Side view of scintillation unit. Notation: 1) 12) are the same as in Figs. 1 and 2;13) amplitude-digital converter AD7888AR; 14) operational amplifier 5442; 15) shifting register (CMOS- based serial-to-parallel code converter) 564IR13.

instead of microstrip detectors, it is supposed to apply newly-developed detectors exploiting the concept of bipolar $n-p-n$ transistor pixels. First high-sensitive matrix-structural detector have been developed and tested. Experimental results correspond to our expectations.

This work is partially supported by Russian Foundation for Basic Research, project no. 05-02-17599-; and Ministry of Education and Science of Russian Federation, project no. LSS 1782.2003.2.

\section{References}

[1] Aleksandrov K.V. et al., Nucl. Phys. B (Proc. Suppl.) 122 (2003) 427; ibid, 113 (2002) 344; Nucl. Instr. Meth. in Phys. Res. A459 (2001) 135. 